\section{BMJ Open}

Ophthalmology

\title{
Post hoc analysis of ellipsoid zone changes beyond the central subfield in symptomatic vitreomacular adhesion patients from the OASIS trial
}

\author{
Swetha Bindu Velaga, ${ }^{1,2}$ Muneeswar Gupta Nittala (D) ,, ${ }^{2,3}$ Michael S Ip, ${ }^{3}$ \\ Luc Duchateau, ${ }^{4}$ SriniVas R Sadda (1) ${ }^{1,3,5}$
}

To cite: Velaga SB, Nittala MG, Ip MS, et al. Post hoc analysis of ellipsoid zone changes beyond the central subfield in symptomatic vitreomacular adhesion patients from the OASIS trial. BMJ Open Ophthalmology 2021;6:e000648. doi:10.1136/ bmjophth-2020-000648

Received 28 October 2020 Accepted 8 April 2021

Check for updates

C) Author(s) (or their employer(s)) 2021. Re-use permitted under CC BY-NC. No commercial re-use. See rights and permissions. Published by BMJ.

${ }^{1}$ Retina, Doheny Eye Institute, Los angeles, California, USA ${ }^{2}$ Ophthalmology, Doheny Eye Institute, University of California Los Angeles, Los Angeles, California, USA

${ }^{3}$ Ophthalmology, University of California Los Angeles David Geffen School of Medicine, Pasadena, California, USA ${ }^{4}$ Biometrics Research Centre, Ghent University, Gent, Belgium ${ }^{5}$ Ophthalmology, University of California - Los Angeles, Los Angeles, California, USA

Correspondence to Dr SriniVas R Sadda; ssadda@ doheny.org

\section{ABSTRACT}

Background/aims OASIS is a Phase IIIb trial (NCT01429441) assessing long-term outcomes in subjects with symptomatic vitreomacular adhesion (VMA). The purpose of this study is to report on the frequency, severity, location and time course of ellipsoid zone (EZ) alterations in ocriplasmin-treated and sham control eyes in the OASIS study.

Methods 220 patients (146 ocriplasmin, 74 sham) subjects with VMA were enrolled in this masked post hoc analysis phase IIlb, randomised, sham-controlled double-masked multicentre clinical trial. A masked post hoc analysis of OCT images was performed at the Doheny Image Reading Center from subjects enrolled in the OASIS trial. The status of the EZ band was assessed in three different macular regions: the central subfield (CS) $(\leq 1 \mathrm{~mm}$ diameter), the parafoveal area (PAA) $(>1$ to $\leq 3 \mathrm{~mm})$ and the perifoveal area (PEA) $(>3$ to $\leq 6 \mathrm{~mm})$. The EZ band was rated as normal/intact, full thickness macular hole (FTMH), abnormal but continuous, discontinuous/disrupted or absent at visits from baseline (pretreatment) to week 1 (day 7), month 1 (day 28), month 3, month 6, month 12 and the final follow-up at month 24 . EZ band status was compared in both study and control eyes.

Results A total of 208 patients (138 ocriplasmin, 70 sham) were included in this analysis. At baseline, FTMH was present in $48.6 \%, 8.0 \%, 0 \%$ and $52.8 \%, 2.9 \%, 0 \%$ in the CS, PAA and PEA of the ocriplasmin and sham groups, respectively. The EZ was graded to be abnormal but continuous, discontinuous/disrupted or absent at Baseline in $21.0 \%, 4.3 \%, 2.8 \%$ in the CS, PAA and PEA, respectively, of the ocriplasmin group; and $12.9 \%, 10.0 \%$, $4.3 \%$ in the CS, PAA and PEA of the sham group. For the ocriplasmin group in the PAA, this frequency increased to $6.6 \%$ at week 1 , was $9.8 \%$ at month 1 , but improved to $3.8 \%$ at month 3 , and remained stable to $1.6 \%$ at month 24. These differences, however, were not statistically significant.

Conclusions Ocriplasmin treatment for symptomatic VMA was associated with EZ abnormalities in a small percentage of patients that was best assessed in regions (PEA) relatively unaffected by the VM interface disease at baseline. The EZ abnormalities were apparent by week 1 , persisted at month 1 , and appeared to resolve in the majority of cases by month 3 .

Trial registration number NCT01429441

\section{Key messages}

What is already known about this subject?

Photoreceptor alterations are known to occur in some patients following ocriplasmin injection, but the frequency and time course of these changes have not been well characterised.

\section{What are the new findings?}

- This analysis from a randomised prospective trial addresses this knowledge gap and demonstrates that these alterations occur in a small minority of patients, are most apparent within one month of treatment, and are largely resolved by 3 months in most cases.

How might these results change the focus of research or clinical practice?

- This study findings may provide insight into the pathophysiology of this process and aid clinicians in counseling patients.

\section{INTRODUCTION}

Persistent vitreomacular adhesion (VMA) can progress to anatomical derangements such as vitreomacular traction (VMT) and/or macular hole $(\mathrm{MH})$. These changes are commonly associated with symptoms such as metamorphopsia, decreased visual acuity and ultimately loss of visual function. ${ }^{1}$ Pars plana vitrectomy with or without internal limiting membrane peeling is the primary therapeutic strategy used to relieve traction and restore visual function loss in the setting of VMT and MH. The development and approval of pharmacological vitreolytic agents has provided clinicians with a non-surgical alternative to treat these conditions. ${ }^{2}$ Ocriplasmin (Jetrea; ThromboGenics, Leuven, Belgium) is an enzyme developed for liquefying the laminin and fibronectin in the vitreous and is administered as a intravitreal injection of $125 \mu \mathrm{g}$ in $0.1 \mathrm{~mL}$ volume dose and is indicated for the treatment of symptomatic VMA including fullthickness (FT) MH. ${ }^{34}$ 
In thephase 3 MIVI-trust pivotal clinical trials, adverse events such as photopsia, metamorphopsia and transient visual acuity loss were frequently reported during the first week after injection in ocriplasmin treated patients, but these problems generally resolved by 1 month after therapy. ${ }^{5}$ During the early postmarketing clinical experience, some cases of acute vision decrease after ocriplasmin therapy were noted concurrent with disruption of the outer retina, and in particular the ellipsoid zone (EZ), as evidenced on spectral domain (SD) OCT. ${ }^{67}$ It should be noted that the original pivotal studies used time domain OCT and thus the EZ may have been more difficult to evaluate. Initial reports suggested that the EZ loss was transient with recovery observed by 28 days in nearly all patients. ${ }^{8}$ Subretinal fluid was noted to also be present in some cases and also resolved by 28 days. ${ }^{8}$ The frequency and time course of changes in the outer retina were not clearly evident from the early reports and small case series.

The Ocriplasmin for Treatment for Symptomatic Vitreomacular Adhesion Including Macular Hole (OASIS) study was a phase 3B randomised clinical trial which was designed to evaluate the long-term ( 24 month) efficacy and safety of ocriplasmin for the treatment of symptomatic VMA, including FTMH. The primary results have been reported previously and showed that VMA resolution at day 28 occurred in a significantly greater proportion of ocriplasmin treated eyes (41.7\%) compared with the sham group $(6.2 \%)$, with persistence of the treatment effect through the duration of the study. In addition, at month $24,50.5 \%$ of ocriplasmin-treated patients had a $\geq 2$ line improvement in best-corrected visual acuity (BCVA) from baseline, compared with $39.1 \%$ in the sham group. FTMH closure (without surgery) was observed in $30.0 \%$ of ocriplasmin eyes compared with $15.4 \%$ in sham eyes. Microperimetry ${ }^{9}$ (MP) and multifocal electroretinography (ERG) data were also obtained in a subset of patients and suggested more stable and central fixation and higher retinal sensitivity in ocriplasmin-treated patients.

The OASIS study included 12 visits over a 2-year period and SD-OCT scans were obtained at each of these visits. Thus, the OASIS study provides a resource to better understand EZ alterations in the setting of symptomatic VMA and ocriplasmin treatment.

The purpose of the present study is to report on the frequency, severity, location, and time course of EZ alterations in ocriplasmin-treated and sham eyes in the OASIS study.

\section{MATERIALS AND METHODS}

The OASIS (NCT01429441) study is a phase IIIb, randomised, sham-controlled double-masked multicentre clinical trial consisting of a total of 220 eyes of 220 patients (146 ocriplasmin, 74 sham) in compliance with the trial protocol and all federal, local, or regional requirements, including with the Health Insurance Portability and Accountability Act. The research also conformed to the tenets of the Declaration of Helsinki. The details of the trial have been extensively described in prior reports. ${ }^{910}$ The present study represents a post hoc analysis of the OCT data from the trial.

Patients aged $\geq 18$ years with the presence of VMA (defined as a central vitreous adhesion to the retina within the $6 \times 6 \mathrm{~mm}$ OCT scan field, surrounded by elevation of the posterior vitreous cortex) which in the investigator's opinion was related to decreased visual function (eg, metamorphopsia, decreased visual acuity, other visual symptom), a BCVA of 20/32 or worse in the study eye, and a BCVA of 20/800 or better in the non-study eye, were eligible for inclusion. Patients were excluded from the study if they had an epiretinal membrane, aphakia, or an $\mathrm{MH}>400 \mu \mathrm{m}$ in diameter, any history of proliferative retinopathy, exudative age-related macular degeneration, retinal vein occlusion, vitreous haemorrhage, uncontrolled glaucoma or any history of vitrectomy in the study eye. Patients who did not achieve vitreous-macular separation could be exited from the study to undergo vitrectomy.

For this post hoc analysis, SD-OCT images (Heidelberg Spectralis OCT; $\left(20^{\circ} \times 20^{\circ}\right.$ volume scans composed of 97 B-scans with 18X averaging); Heidelberg, Germany) from subjects enrolled in the OASIS trial were transferred to the Doheny Image Reading Centre for assessment. OCT data was assessed at Baseline (pretreatment), and week 1 (day 7), month 1 (day 28), month 3, month 6, month 12

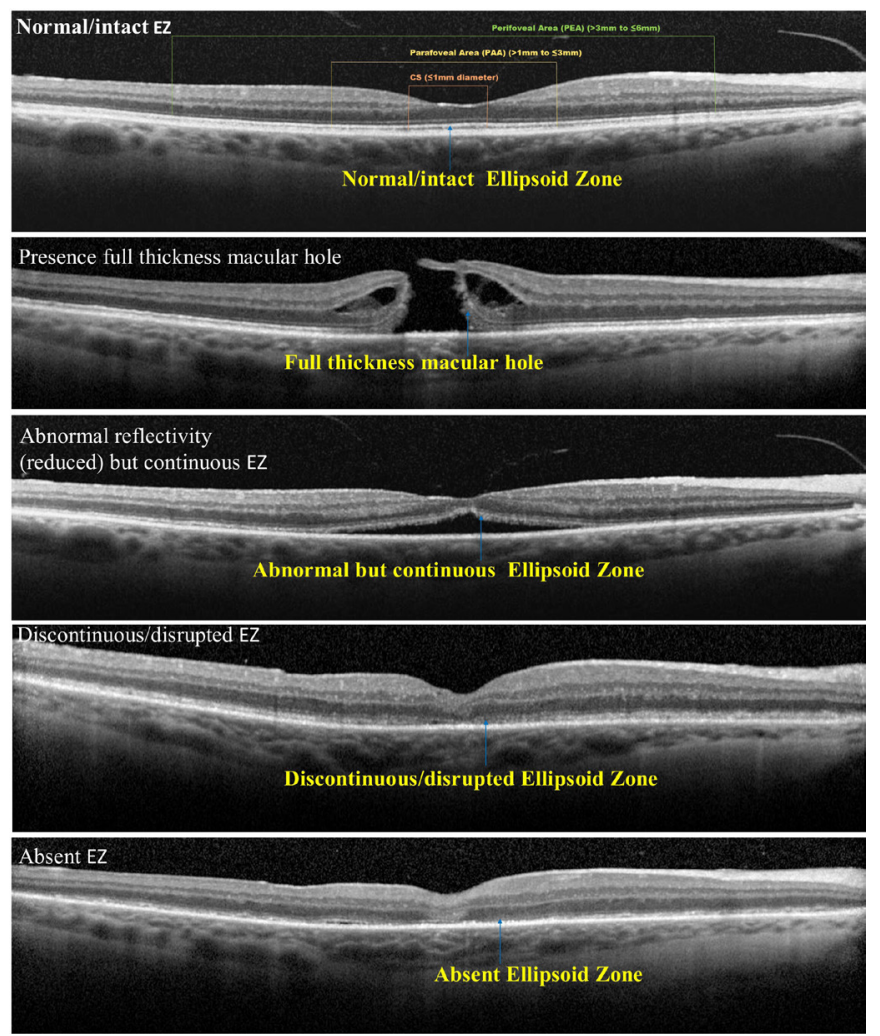

Figure 1 Grading scale for EZ band classification. Normal/ intact and four categories of abnormal EZ: presence of full thickness macular hole; abnormal but continuous; discontinuous/disrupted and absent. EZ, ellipsoid zone. 
Table 1 The baseline demographic characteristics between the two study arms

\begin{tabular}{|c|c|c|c|c|}
\hline & Total study cohort & Sham study cohort & Ocriplasmin study cohort & $P$ value \\
\hline \multicolumn{5}{|l|}{ Age (years) } \\
\hline N (eyes) & 208 & 70 & 138 & \\
\hline Mean (SD) & 69.25 (10.3) & $68.36(10.94)$ & 69.7 (9.98) & 0.38 \\
\hline Minimum, maximum & 38,94 & 39,89 & 38,94 & \\
\hline \multicolumn{5}{|l|}{ Gender } \\
\hline N (patients) & 208 & 70 & 138 & \\
\hline Male (\%) & $69(33.17)$ & $26(37.14)$ & $43(31.16)$ & \\
\hline Female (\%) & $139(66.83)$ & $44(62.86)$ & $95(68.84)$ & 0.49 \\
\hline \multicolumn{5}{|l|}{ Lens status } \\
\hline $\mathrm{N}$ (patients) & 208 & 70 & 138 & \\
\hline Phakic & $151(72.6)$ & $50(71.43)$ & 101 (73.19) & \\
\hline Pseudophakic & $57(27.4)$ & $20(28.57)$ & $37(26.81)$ & 0.92 \\
\hline
\end{tabular}

and month 24 after treatment by certified OCT graders ( $\mathrm{SV}$ and $\mathrm{MN}$ ), who reviewed all B-scans in the volume. The status or integrity of the EZ band was assessed in three different macular regions: the Central Subfield (CS) (central one $1 \mathrm{~mm}$ diameter zone), the parafoveal area (PAA) (ring extending from $>1$ to $\leq 3 \mathrm{~mm}$ ) and the perifoveal area (PEA) (ring extending from $>3$ to $\leq 6 \mathrm{~mm}$ ). Loss of integrity of the EZ band in these regions were described, in terms of severity, as abnormal but continuous EZ, discontinuous/disrupted EZ and absent EZ. A FTMH may represent a dehiscence and separation of the retina rather than a true loss of the photoreceptors but for this analysis was also considered to be one of the four pre-specified categories of abnormal EZ band (figure 1). This generally impacted the CS and to a lesser extent the
PAA. For comparisons between ocriplasmin and sham groups, FTMH were considered separate from the other three abnormal EZ categories which were combined for these comparisons. All OCT images were graded in a masked fashion (masked as to treatment assignment and visit order) by one senior OCT grader (SV). In addition, $10 \%$ (197 visits) of all cases/visits were randomly selected and assessed by a second masked senior grader to assess the reproducibility of the grading.

The ocriplasmin and sham groups were compared at the different time points with respect to the presence of abnormal EZ band by exact logistic regression at the three locations (central, parafoveal, perifoveal) separately at the $5 \%$ significance level. Both the percentage abnormalities and the OR with its $95 \%$ CI are used as summary

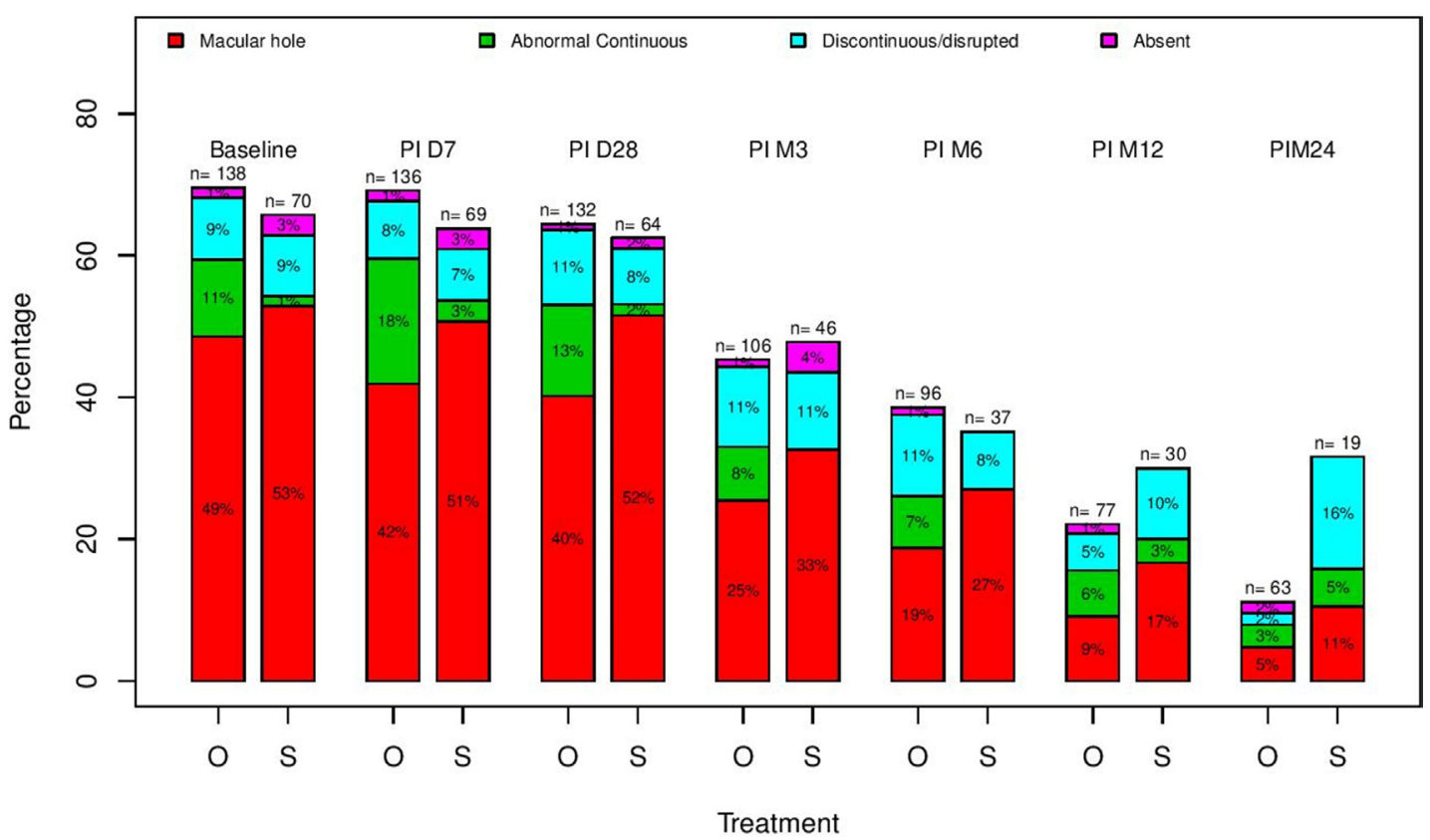

Figure 2 Percentage of abnormalities of the EZ in the central subfield at baseline, postinjection day 7, day 28, month 3 , month 6 and month 24 in both study cohorts. Only the categories of abnormal EZ with the percentages given relative to the entire cohort (including eyes with normal/intact EZ; O-Ocriplasmin cohort, S-Sham cohort). EZ, ellipsoid zone. 


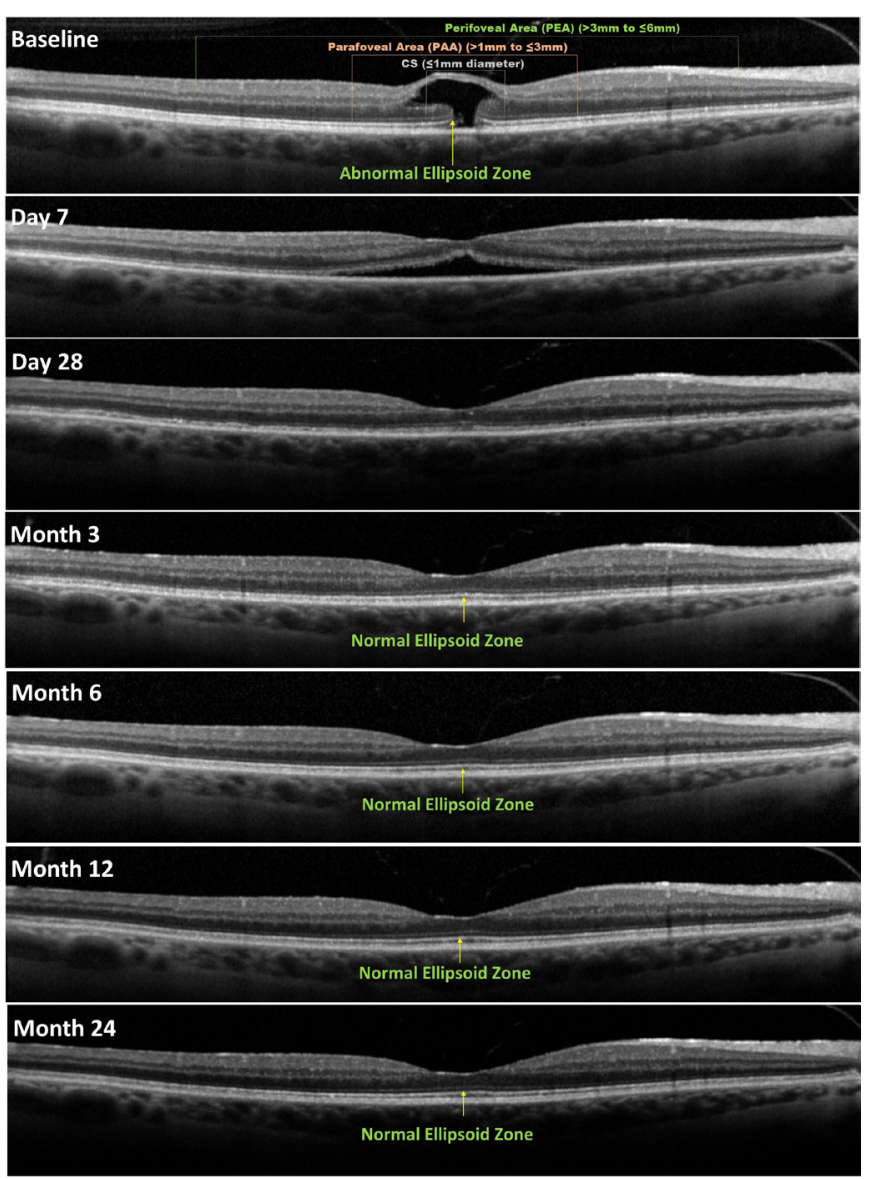

Figure 3 Representative SD-OCT image of a patient with disrupted $\mathrm{EZ}$ at baseline and with subsequent restoration by month 3 following ocriplasmin injection. CS, central ubfield; EZ, ellipsoid zone; SD, spectral domain .

statistics. These analyses were performed for the whole group of patients, but also for the subgroup of patients with and without abnormal EZ band at baseline. The reproducibility of the grading was assessed by weighted kappa statistic using the criteria of Landis and Koch. Age between the two treatment groups was compared using the t-test, and frequency of gender and lens status categories by the $\chi^{2}$ test with Yates correction. All statistical analyses of the data were performed using commercial software SAS (V.9.4; SAS Institute).

\section{RESULTS}

Of the total of 220 patients, 208 patients (208 eyes; 138 ocriplasmin, 70 sham) were included in this analysis because for 10 patients no baseline value was available and for 2 patients only the baseline value was available. The mean age of the overall cohort was $69.25 \pm 10.3$ (median 68; range 38-94) years and $33.2 \%$ were male. The baseline demographic characteristics were similar between the two study arms (table 1). Notably, FTMH were present in $34.1 \%$ of ocriplasmin and $37.1 \%$ of sham eyes at baseline. At baseline, subretinal fluid was present in $37.7 \%(52 / 138)$ patients in the ocriplasmin group and $42.9 \%(30 / 70)$ patients in the sham group. The sample size for analysis decreased over time in both groups partially due to the fact that patients with persistent vitreo-macular traction could be exited from the study to undergo vitrectomy, after which event their data were no longer used in the analysis.

\section{CS assessment}

At baseline, the EZ was abnormal in $69.6 \%$ of eyes in the ocriplasmin arm and in $65.7 \%$ of eyes in the sham arm, with $48.6 \%$ and $52.8 \%$ of the eyes presenting with FTMH in the ocriplasmin and sham arm respectively and $21.0 \%$ and $12.9 \%$ of the eyes presenting with abnormal but continuous, discontinuous/disrupted, or absent EZ in the ocriplasmin and sham arm respectively (figure 2).

The presence of abnormal but continuous, discontinuous/disrupted or absent EZ in the ocriplasmin group increased to $27.2 \%$ at week 1 but decreased to $19.8 \%$ at month 3 and to $6.4 \%$ at month 24 .

At month 24, the normalisation of the $\mathrm{EZ}$ band in the CS was more frequent in the ocriplasmin-treated group (88.9\%; 95\% CI 78.4 to 95.4) compared with sham (68.4\%; 95\% CI 43.4 to 87.4), but the difference was not statistically significant $(\mathrm{p}=0.085)$.

The only significant effect was found for the subgroup of patients that started with a normal EZ, who demonstrated a significantly higher frequency of abnormal EZ at week 1 in the ocriplasmin group compared with the sham group $(\mathrm{OR}=6.02, \mathrm{p}=0.039)$. This difference resolved quickly and reversed at month 24 , with a numerically higher frequency in the sham group compared with the ocriplasmin group $(\mathrm{OR}=0.40, \mathrm{p}=0.286)$. A representative case is shown in figure 3 .

\section{PAA assessment}

At baseline, $8.0 \%$ and $2.9 \%$ of the eyes were noted to have a FTMH involving the PAA region in the ocriplasmin and sham arm respectively. $4.3 \%$ and $10.0 \%$ of the eyes presented with abnormal but continuous, discontinuous/disrupted, or absent EZ at baseline in the ocriplasmin and sham arm, respectively (figure 4).

The presence of abnormal but continuous, discontinuous/disrupted, or absent EZ in the ocriplasmin group increased to $9.5 \%$ at week 1 but decreased to $3.7 \%$ at month 3 and to $2.6 \%$ at month 24 .

At month 24, the normalisation of the EZ band in the PAA zone was more frequent in the ocriplasmin-treated group (96.8\%; 95\% CI 89.0 to 99.6) compared with sham (84.2\%; 95\% CI 60.4 to 96.6$)$, but the difference was not statistically significant $(\mathrm{p}=0.600)$.

No significant differences were found at any time or in any of the subgroups.

\section{PEA assessment}

At baseline, none of the eyes demonstrated evidence of FTMH in the PAA region (figure 5), whereas $2.8 \%$ and $4.3 \%$ of the eyes presented with an abnormal but continuous, discontinuous/disrupted, or absent EZ at baseline in the ocriplasmin and sham arm, respectively. 




Figure 4 Percentage of abnormalities of the EZ in the parafoveal area at baseline, post injection day 7, day 28, month 3 , month 6 and month 24 in both study cohorts. Only the categories of abnormal EZ with the percentages given relative to the entire cohort (including eyes with normal/intact EZ; O-ocriplasmin cohort, S-Sham cohort). EZ, ellipsoid zone.

The presence of abnormal EZ in the ocriplasmin group increased to $6.6 \%$ at week 1 but decreased to $3.8 \%$ at month 3 and to $1.6 \%$ at month 24 .

At month 24, the normalisation of the EZ band in the PEA zone was more frequent in the ocriplasmin-treated group (96.8\%; $95 \%$ CI 89.0 to 99.6) compared with sham (94.7\%; 95\% CI 74.0 to 99.9$)$, but the difference was not statistically significant $(\mathrm{p}=1.000)$.

No significant differences were found at any time or in any of the subgroups.

\section{Reproducibility analysis}

The agreement between graders for EZ assessment using SD-OCT images was excellent with kappa value of 0.91 for CS area, 0.91 for PAA and 0.99 for PEA.

\section{DISCUSSION}

In this post hoc analysis, we report the integrity of the EZ in subjects enrolled in the OASIS trial over time after ocriplasmin or sham injection. We observed that while an increase in the severity of EZ abnormalities may be

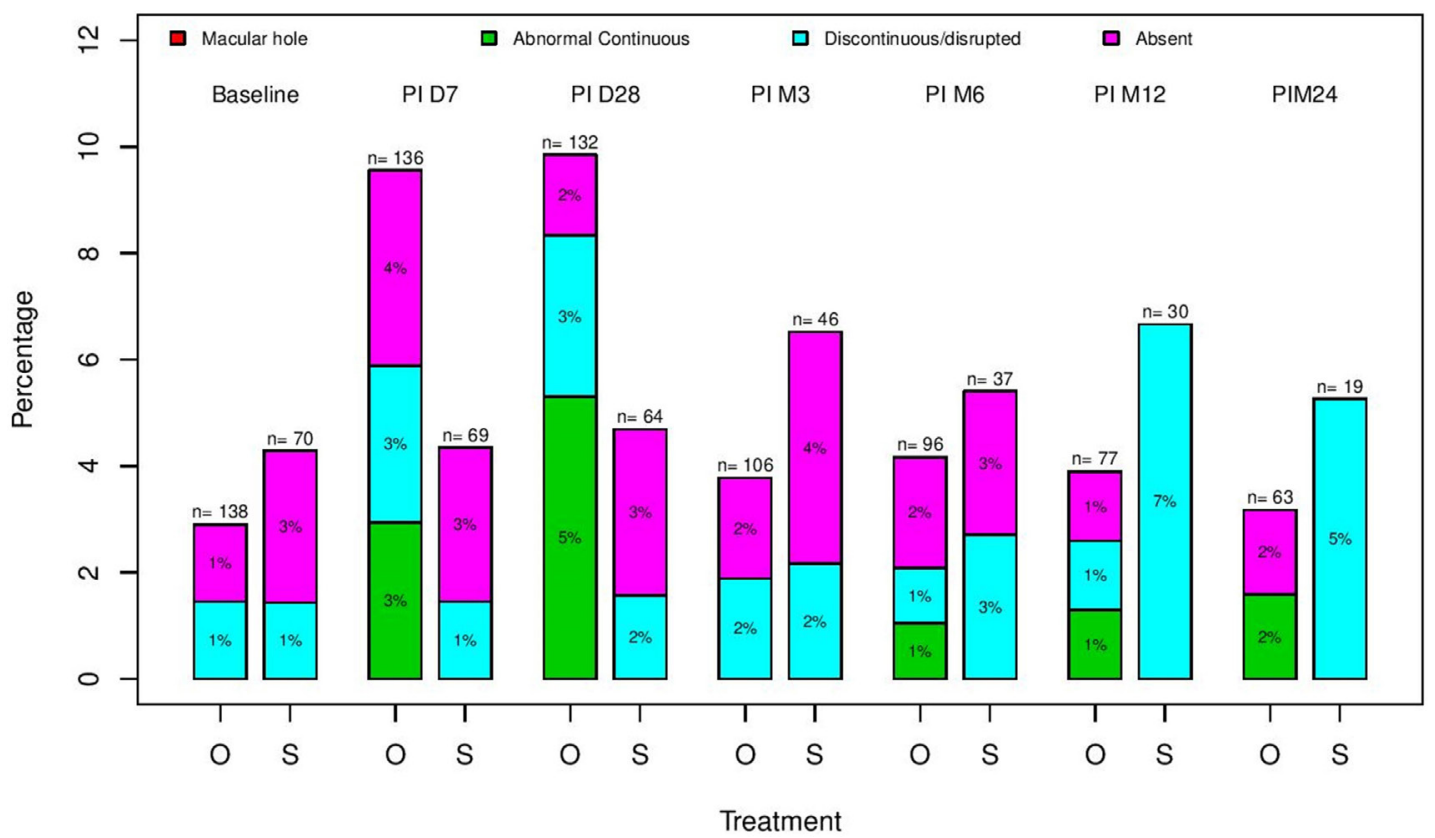

Figure 5 Percentage of abnormalities of the EZ in the perifoveal area at baseline, postinjection day 1, day 28, month 3, month 6 and month 24 in both study cohorts. Only the categories of abnormal EZ with the percentages given relative to the entire cohort (including eyes with normal/intact EZ; O-Ocriplasmin cohort, S-Sham cohort). EZ, ellipsoid zone. 
observed at week 1 and month 1 after treatment, this abnormality appears to resolve in nearly all subjects by month 3 and this was sustained out to month 24 .

A number of previous smaller studies have assessed the EZ in the setting of ocriplasmin. Tibbets $e t a l^{11}$ demonstrated a disruption of the EZ associated with reduced ERG amplitudes with symptoms of darkening of the vision that persisted for at least 4 months. Margo et $a l^{12}$ reported EZ and ERG abnormalities that persisted for 14 months after ocriplasmin treatment. Tschuppert S et al reported on a series of 12 consecutive patients with VMT treated with ocriplasmin, and observed subfoveal fluid after injection in 9 of these cases which was associated with vision loss, but resolved by 6 weeks in 8 of the 9 cases. ${ }^{13}$ Freund et $a l^{14}$ reported EZ disruption 1 week after treatment of an FTMH with ocriplasmin, but already noted some recovery of the EZ by 3 weeks. Cereda $e t a l^{15}$ used widefield OCT $\left(55^{\circ}\right)$ to better evaluate the extent of EZ changes and observed evidence of EZ attenuation throughout the scanning field (they termed 'panretinal') in 8 of 15 eyes, with recovery of the photoreceptors in 7 of 8 eyes. Itoh $e t a l^{67}$ used an ellipsoid mapping technique and observed subretinal fluid and EZ attenuation in 7 of 19 treated patients at 1 week with recovery of the EZ by 3 months. The ASRS Therapeutic Surveillance Committee also summarised initial postmarketing experience with ocriplasmin but described only eight cases $(0.18 \%$ of cases) with EZ alterations, of which at least six resolved. ${ }^{16}$

The various studies have highlighted that the EZ may be impacted by ocriplasmin in some cases, but as they were small retrospective series or case reports or incomplete post-marketing surveys, it was difficult to establish the true frequency and time course of the problem. The prospective Phase 3b OASIS trial offered an opportunity to study this problem more precisely, in greater detail and with use of available SD-OCT. Previously, we reported on a substudy of subjects from the OASIS trial who underwent MP to better understand the impact on visual function. ${ }^{9}$ This MP sub study only included 27 subjects, but demonstrated a consistently better sensitivity in ocriplasmin treatment patients. However, a transient reduction in sensitivity in the ocriplasmin group was noted at week 1 and month 1 . The previous analysis, however, did not focus on the OCT data to see if it demonstrated a similar pattern. Unlike the MP data, the OCT data were available for the entire cohort and was the subject of the analysis in this report.

Overall, the anatomic EZ analysis from the OCT appeared to parallel the previous MP findings. In particular, a transient increase in EZ disruption/abnormality was observed by 1 week after ocriplasmin treatment in a small percentage of subjects and this abnormality appeared to persist through month 1 , but had returned to approximately baseline by month three and remained stable through the final assessment at month 24. This general pattern was apparent in all zones (CS, PAA, PEA), though it was more difficult to discern in the CS. This is not surprising since the CS, and to a lesser extent the PAA, could be directly involved by an FTMH; or in the absence of FTMH, and were more likely to be significantly impacted by the vitreomacular traction process. Although the vitreous adhesion was present on the epiretinal surface, the tractional effects could impact the entire thickness of the retina. This is readily apparent, when one considers the frequency of abnormal or discontinuous EZ in CS at baseline in both the ocriplasmin and sham groups. The baseline presence of EZ abnormality is a potential confounder in our understanding of the impact of ocriplasmin. The perifoveal region (PEA), in contrast, was relatively normal at baseline-in fact the EZ was assessed to be completely normal in this region in 96\%-97\% of cases. Thus, the PEA may be best location to evaluate for the potential direct effect of ocriplasmin treatment on the EZ. In the PEA, an additional 7 percent of subjects developed EZ abnormalities at week 1 . The same frequency was observed at month 1 , and by month 3, the frequency of EZ abnormality had returned nearly to the baseline level.

Overall, our findings would seem to highlight that EZ alterations only occur in a minority of ocriplasmin patients and that in vast majority of these patients the alterations resolve over time, typically by 3 months. These findings are very much in line with previously reported electrophysiologic and microperimetric findings. ${ }^{9}$

Our study is not without limitations, including most notably the loss of subjects over time which was by virtue of the design of the study which permitted subjects who did not experience release of traction to be exited and undergo vitrectomy or other procedures per their treating physician. The loss of subjects was especially problematic in the sham group where most patients did not demonstrate a spontaneous release. Because of this loss to follow-up, the comparisons between ocriplasmin and sham patients might be confounded. Another limitation is that the EZ was assessed qualitatively and categorically which precludes a more granular assessment of the time course of recovery. The EZ was also assessed on a single horizontal B-scan. While it is possible this may have missed localised EZ abnormalities in other sectors, that situation is probably unlikely based on Cereda's widefield OCT analysis which showed diffuse alterations throughout the retina in affected eyes. ${ }^{15}$ An en face EZ mapping approach as described by Itoh et al may be more informative, but this would require dense OCT scanning. In addition, as the EZ reflectivity was assessed qualitatively, it is unknown whether more subtle reflectivity abnormalities may have been present in eyes with an otherwise normal appearing EZ. Lastly, although we do show anatomic improvement of the EZ following treatment with ocriplasmin, functional assessment (eg, MP or ERG assessment) to correlate with the anatomic changes would have been of interest, but this was not available.

An important strength of this study is the use of experienced masked reading centre graders to assess the EZ. Thus, compared with previous studies, we believe the present study provides greater insight into the true 
frequency, severity and durability of the impact of ocriplasmin on the outer retina. Additionally, as compared with previous studies on this topic, SD-OCT (performed by certified evaluators) was available for evaluation.

In summary, in the OASIS study, ocriplasmin treatment was associated with a transient disruption of the EZ in $<10 \%$ of subjects that was evident by 1 week after treatment and recovered in nearly all subjects by 3 months after treatment. This recovery was sustained out to 24 months.

Contributors SV: collecting the data, analysing the data and writing the manuscript. MN: collecting the data, analysing the data and review the manuscript. MSI: critical review. LD: statistical analysis. SRS: study design and critical review of the manuscript.

Funding The authors have not declared a specific grant for this research from any funding agency in the public, commercial or not-for-profit sectors.

Competing interests None declared.

Patient and public involvement Patients and/or the public were not involved in the design, or conduct, or reporting, or dissemination plans of this research.

Patient consent for publication Not required.

Ethics approval All study grading procedures were approved by the institutional review board at University of California Los Angeles (number: 15-000083).

Provenance and peer review Not commissioned; externally peer reviewed.

Data availability statement Data are available upon request.

Open access This is an open access article distributed in accordance with the Creative Commons Attribution Non Commercial (CC BY-NC 4.0) license, which permits others to distribute, remix, adapt, build upon this work non-commercially, and license their derivative works on different terms, provided the original work is properly cited, appropriate credit is given, any changes made indicated, and the use is non-commercial. See: http://creativecommons.org/licenses/by-nc/4.0/.

ORCID IDs

Muneeswar Gupta Nittala http://orcid.org/0000-0002-3802-6594

SriniVas R Sadda http://orcid.org/0000-0001-8504-8051

\section{REFERENCES}

1 Jackson TL, Regillo CD, Girach A, et al. Baseline predictors of Vitreomacular Adhesion/Traction resolution following an intravitreal injection of Ocriplasmin. Ophthalmic Surg Lasers Imaging Retina 2016;47:716-23.

2 Lescrauwaet B, Duchateau L, Verstraeten T, et al. Visual function response to ocriplasmin for the treatment of vitreomacular traction and macular hole: the OASIS study. Invest Ophthalmol Vis Sci 2017;58:5842-8.

3 Chen W, Mo W, Sun K, et al. Microplasmin degrades fibronectin and laminin at vitreoretinal interface and outer retina during enzymatic vitrectomy. Curr Eye Res 2009;34:1057-64.

4 Schumann RG, Langer J, Compera D, et al. Assessment of intravitreal ocriplasmin treatment for vitreomacular traction in clinical practice. Graefes Arch Clin Exp Ophthalmol 2017;255:2081-9.

5 Stalmans P, Benz MS, Gandorfer A, et al. Enzymatic vitreolysis with ocriplasmin for vitreomacular traction and macular holes. $N$ Engl $J$ Med 2012;367:606-15.

6 Itoh Y, Vasanji A, Ehlers JP. Volumetric ellipsoid zone mapping for enhanced visualisation of outer retinal integrity with optical coherence tomography. Br J Ophthalmol 2016;100:295-9.

7 Itoh Y, Kaiser PK, Singh RP, et al. Assessment of retinal alterations after intravitreal ocriplasmin with spectral-domain optical coherence tomography. Ophthalmology 2014;121:2506-7.

8 Singh RP, Li A, Bedi R, et al. Anatomical and visual outcomes following ocriplasmin treatment for symptomatic vitreomacular traction syndrome. Br J Ophthalmol 2014;98:356-60.

9 Sadda SR, Dugel PU, Gonzalez VH, et al. The OASIS MP-1 substudy: characterization of the effect of Ocriplasmin on microperimetry parameters. Retina 2019;39:319-30.

10 Dugel PU, Tolentino M, Feiner L, et al. Results of the 2-year Ocriplasmin for treatment for symptomatic Vitreomacular adhesion including macular hole (OASIS) randomized trial. Ophthalmology 2016;123:2232-47.

11 Tibbetts MD, Reichel E, Witkin AJ. Vision loss after intravitreal ocriplasmin: correlation of spectral-domain optical coherence tomography and electroretinography. JAMA Ophthalmol 2014;132:487-90.

12 Margo JA, Schocket LS, Klima K, et al. Persistent retinal changes after intravitreal ocriplasmin. Retin Cases Brief Rep 2016;10:48-51.

13 Tschuppert S, Gerding H. Transient subfoveal fluid and visual loss after Ocriplasmin. Klin Monbl Augenheilkd 2016;233:453-5.

14 Freund KB, Shah SA, Shah VP. Correlation of transient vision loss with outer retinal disruption following intravitreal ocriplasmin. Eye 2013;27:773-4.

15 Cereda MG, Corvi F, Cozzi M, et al. Optical coherence tomography 2: diagnostic tool to study peripheral vitreoretinal pathologies. Retina 2019;39:415-21.

16 Hahn P, Chung MM, Flynn HW, et al. Safety profile of ocriplasmin for symptomatic vitreomacular adhesion: a comprehensive analysis of premarketing and postmarketing experiences. Retina 2015;35:1128-34. 